\title{
Projektionen. Entwürfe einer Welt von morgen im internationalen Spielfilm
}

\author{
von Reinhold Jacobi
}

Filme sind einerseits Projektionen von Bildern auf eine Leinwand oder einen Bildschirm, andererseits aber auch Projektionen „in Bildern”, die Ausdruck sind von Erwartungen oder Befürchtungen, Hoffnungen oder Ängsten der Menschen. Spielfilme und nur dieses zugegebenermaßen sehr eingeschränkte Verständnis von Film soll dieser Betrachtung des Mediums zugrunde liegen - stellen nicht selten mehr oder minder umfängliche Entwürfe von Welt vor, die eben als konstruiert erscheint aus Produkten von Hoffnung oder Furcht. Insofern zeichnet das Medium Film eine ganz eigenartige, aber besonders prägnante Dimension aus, die mit dem Begriff des „Visionären" angedeutet werden kann.

Im Verständnis der meisten Zuschauer und der Majorität derjenigen, die mit diesem Medium im Herstellungsprozeß zu tun haben, gilt der Spielfilm vorrangig als ein Mittel der Unterhaltung. Diese Funktion war zumindest in den ersten Jahrzehnten der Filmgeschichte absolut vorrangig. Auch heute kann man davon ausgehen, daß Unterhaltung im Sinne der Ablenkung, Zerstreuung und Spannung maßgebliches Ziel des (Kino-)Films ist. Von der technischen Seite aus betrachtet, versteht sich der Film grundsätzlich als Seh-Medium, Augen-Medium, weniger als Hör-oder Ohren-Medium, auch wenn letztere Komponente unabdingbar zum heute allgemein üblichen Ton-Film hinzugehört. Film ohne „visionäre” Dimension wäre insofern ein Widerspruch in sich. Da ein solcher nicht beabsichtigt ist, meint ,visionäre Dimension" des Films eine zusätzliche, wichtige inhaltliche Komponente, die über das schier Optische hinausweist.

Gemeinhin wird mit Vision eine geistige Schau gemeint, eine Vorstellung in physisch nicht existenten Bildern, die dem einzelnen, der zu einer solchen geistigen Schau fähig ist, zugeordnet werden kann. Der Künstler wird sein Werk auf solche ,geistige Schau" beziehen, für die allerdings der Begriff "Vision” in der Regel als zu ambitioniert, als zu pathetisch abgelehnt werden wird. Eine Erweiterung erfährt „Vision”, durch die nicht unbedingt auf Zukunft verwiesen wird, durch den abgeleiteten Begriff des Visionären. Spricht man vom Visionären, so meint man das Erscheinen beziehungsweise Schauen von Dimensionen der Zukünftigkeit. Gleichzeitig wird darunter eine eher vieldeutige, komplexe Erfahrung verstanden werden müssen, die dem Optischen viel verdankt.

In diesem Sinne bezeichnet das Visionäre eine ganz entscheidende Dimension des Spielfilms. Aufgrund der technischen, apparativen, reproduktiven Möglichkeiten des Mediums wird durch dieses nicht nur Wirklichkeit im Sinne von Gegenwärtigkeit abgebildet, sondern diese wird häufig überschritten in Richtung auf nicht unmittelbar zugängliche räumliche und insbesondere zeitliche Dimensionen hin. Seiner Fähigkeit, in visionärer Weise Zukünftiges zu gestalten, verdankt der Film ein hohes Maß an Faszinationsvermögen. Er nimmt nämlich dem Zuschauer die Mühe der geistigen Vorstellungsverdichtung - im Unterschied etwa zur erzählenden Literatur - weithin ab, wird dadurch allerdings nicht selten auch eindimensional, „ärmlich" und dadurch fragwürdig gegenüber dem subjektiven Phantasiepotential eines jeden Zuschauers.

Dr. Reinhold Jacobi ist Leiter des Referats Film/AV-Medien in der Zentralstelle Medien der Deutschen Bischofskonferenz, Bonn. 
Dieses Úbernehmen einer speziellen Mühe bedeutet jedenfalls eine Anregung, ein Angebot, einer bestimmten Vorstellung zu folgen. Zukünftiges entwerfen zu können und dies mit der dem Film ureigenen Möglichkeit technischer Perfektion zu leisten, gibt dem Film im Vergleich zu den anderen Künsten einen besonderen Rang.

Andererseits bedeutet das Visionäre aller Kunst zunächst „Vorstellung” im Sinne von Vorstellungsvermögen und Vorgestelltem. Hierbei gewinnt das Zukünftige, das in die Zukunft Projizierte, das dann auch möglicherweise Utopische gar keine besondere Bedeutung. Kunst ist immer geistige Vorstellung, die Gestalt gewonnen und Form angenommen hat. Insofern kann auch die Vorstellung von Vergangenem und von Vergangenheit eine Angelegenheit von visionärem Rang sein. So ist bekanntermaßen auch die Gestaltung von Gegenwart dazu geeignet, ein Ergebnis visionärer Kraft zu sein und eine visionäre Leistung. Im übertragenen Sinne und auf den Film bezogen, bedeutet dies, da z.B. die von der „visionären Dimension” gemeinten Inhalte beziehungsweise Stoffe nicht vorrangig etwa im ganz zukünftig gemeinten Science-Fiction-Film aufgehoben sein müssen. Filmstoffe, die in der Gegenwart angesiedelt sind, taugen zur Eröffnung von Perspektiven in die Zukunft unter Umständen erheblich mehr.

Künstlerische und geistige Projektion im Sinne des Visionären hat es in jedem Falle mit Entwurf zu tun, hat zu tun mit der Vorstellung von Zukünftigem und von Gegenwärtigem als derjenigen Kategorie, die immer Zukünftiges in sich trägt. Wenn Welt von morgen im technisch-künstlerischen Medium Film entworfen wird, dann geht es um Hoffnungen und Erwartungen, Befürchtungen und Ängste, um Vermutungen und Prophezeiungen, nicht selten auch um Drohungen. Wenn Zukunft angegangen wird, dann kann es um die Vorstellung von Paradies, von Utopia, nicht selten von Schlaraffenland, aber auch um die Vorstellung von Zusammenbruch und Untergang gehen. Erlösung und Verderben sind die eschatologischen Extreme, um die eben auch künstlerische Phantasie kreist. Nur muß ein auf Zukunft gerichtetes Interesse so weit nicht gehen, tut dies gewöhnlich auch nicht. Da die Tiefendimension von Zukunft unbeschränkt ist, genügt zunächst die Frage nach der allernächsten Zukunft, nach derjenigen etwa der nächsten Generation. Entwürfe einer Welt von morgen bemühen sich daher häufig um die Frage nach der Jugend, indem sie die Welt des heutigen Menschen auf Lebensfähigkeit und Lebenswert für diejenigen befragen, die diese Welt noch nicht gestalten können.

Wenn im folgenden danach gefragt wird, was Film in diesen Jahren mit dem skizzierten Potential an visionärer Perspektiventwicklung auf Zukunft hin anfängt, dann können nur einige Elemente bruchstückhaft benannt werden. Eine derartige Bemühung bezieht sich auch nicht auf die Kenntnis der breiten Masse der in den letzten Jahren angebotenen Kinofilme, sie kann es schlechterdings auch nicht angesichts der Tatsache, da $B$ allein Indien etwa 600 Filme im Jahr produziert. Die Rede kann also nur sein von verhältnismäßig gängigen, zugänglichen und geografisch sehr weit verbreiteten Beispielen. Der außerordentliche Erfolg solcher Filme läßt Rückschlüsse auf die Affinität der Themen zum geografisch nicht gebundenen Publikumsinteresse zu.

Im Vordergrund des Angebots von Filmen mit globalen Aussagen über zukünftige Weltund Lebensbefunde stehen Filme aus den USA und aus Westeuropa. Deren internationale Verbreitung ist relativ groß, vergleicht man sie mit der Verbreitung von Filmen der übrigen Kontinente. Filmische Zukunftsentwürfe der genannten Bereiche sind auch des- 
wegen eher greifbar, weil hier nach wie vor und unbeschadet temporärer oder regionaler Schwierigkeiten umfängliche und vielseitige Filmproduktion möglich ist, nicht zuletzt wegen ihrer Zubringerfunktion gegenüber dem Fernsehen. Der afrikanische Film etwa ist gegenwärtig dabei, sich aus vielfältigen, aber sehr vereinzelten nationalen und regionalen Anfängen heraus zu formieren. Abgesehen davon, daß die Filmländer der westlichen Hemisphäre vom asiatischen Film nurverschwindend geringe Kenntnisse erhalten, obwohl die Produktion von Indien - wie erwähnt - , Hongkong oder Japan enormen Umfang hat, ist bekannt, daß das überwältigende Gros dieser Produktionen von traditionellen Erzählstrukturen auf Serie getrimmter Unterhaltungskonfektion bestimmt ist, die weithin zudem Stoffen regionaler beziehungsweise nationaler Vergangenheit abgewonnen sind - unbeschadet einiger verhältnismäßig weniger ambitionierter und alternativer Filme. Die kleinen asiatischen Länder bauen erst - und dieses, wie am Beispiel derPhilippinen etwa abzulesen ist, mit erstaunlicher Begabung und Vitalität - an Grundlagen nationaler filmischer Bemühung. Die lateinamerikanischen Länder, die eine höchst fruchtbare Phase eines Kinos der sozialen Anklage und des nicht selten revolutionären Appells an die Kräfte der Veränderung in den Fünfziger- und Sechziger-Jahren hinter sich haben, differenzieren unter zum Teil großen Mühen nationale Filmproduktionen aus, wobei international ansprechende Qualität eine spezifische Schwierigkeit darstellt ${ }^{1}$. Der Ostblock schließlich kultiviert ein Kino der Alltagsbewältigung und dergesellschaftlichen Stabilisierungsbemühung mit Hilfe der filmischen Erinnerung an die in der Historie schon aufspürbaren nationalen Qualitäten. Allerdings gelingen dabei immer wieder Beispiele hoher formaler und stofflicher Qualität, denen auch visionäre Dimensionen nicht fremd sind. Leider erreichen die wichtigsten Filme, die internationale Vergleiche nicht nur aushalten, sondern bereichern, nur selten die westliche Welt ${ }^{2}$.

III

Eine derartige pauschale Inblicknahme inhaltlicher filmischer Bemühungen in den verschiedenen Hemisphären drängt den pointierten Schluß auf, sich mit Zukunftsentwürfen, mit dem filmisch ausformuliertem Interesse an der Welt von morgen beschäftigen zu können, sei eine Art Privileg Europas und Nordamerikas. Ein solcher Eindruck täuscht insofern nicht, als es in einem entscheidenden Bereich der Beschäftigung mit Zukunft ein enormes Übergewicht von Produktionen aus den USA gibt, die hier eine Art Domäne haben: beim Science-Fiction-Film ${ }^{3}$. Amerikanische Pioniermentalität und eine ausgesprochene Vorrangstellung auf den Gebieten naturwissenschaftlicher und technologischer Forschung und Entwicklung haben diesem Genre Vorschub geleistet. Meistens interessierte bisher die abenteuerliche Spannungskomponente, wenn etwa durch chemische oder physikalische Unfälle Mutationen von Lebewesen auftraten, die wiederum die Menschheit bedrohten, oder wenn in Fortschreibung von gängigen Lebensformen futurologische Visionen von einem gänzlich mechanisiert-verwalteten Menschendasein im Sinne von Orwells „1984” entworfen wurden. Kritische und satirische Töne haben bei solchen Stoffen gerade in den letzten Jahren zugenommen ${ }^{4}$.

Eine Welle von Filmen entstand geradezu über Stoffe, die im Weltraum angesiedelt werden. Die insbesondere von Amerikanern und Russen konkretisierte Utopie der Weltraumfahrt zu Mond und anderen Planeten hat einen Phantasieschub bewirkt, der in zum Teil spektakulären Produktionen Niederschlag fand. Stanley Kubricks „2001 - Odyssee im Weltraum” $(1968)^{5}$ hat in diesem Genre Maßstäbe gestzt. Nicht nur wird ein perfektes und in seiner Differenziertheit außerordentlich plausibles System raumfahrerischer Fluggeräte vorgeführt, was allein schon, vom Standpunkt der Filmausstattung und Tricktechnik her, eine bemerkenswerte Leistung darstellt. Es wird der Reiz am Apparativen 
durch den Versuch einer menschheitsgeschichtlich-philosophisch eingefärbten Fragestellung schon weit zurückgelassen. Kubrick erinnert dabei an die Urmenschen, von denen einer einen jener Monolithen entdeckt, mit denen seine Nachfahren Jahrmillionen später - eben im Jahre 2001 - im Weltraum konfrontiert werden. Dies ist der ins Bild gebrachte Versuch einer Reflexion über die schwer darstellbare Entwicklungsspanne des Menschen, dem es letztlich um das geistige Prinzip (,Stein des Weisen”?) geht. Derselbe Mensch, der den Quader berührte, entdeckt die Funktionsfähigkeit eines Knochens, den erals Schlagwerkzeug gebrauchen kann; als er ihn in die Luft wirft, verwandelt er sich in ein Raumflugzeug. Später dann rast das Raumschiff in einer (farbfilmisch phantastisch gestalteten) Fahrt immertieferin den unendlichen Raum, einer Fahrt, die jäh endet durch die Erinnerung des Raumfahrers an seine eigene Alterung. Das „geistige Prinzip”, so stellt sich am Ende heraus, ist durch die technologische Intelligenz bestimmt: Von der Menschheitsgeschichte interessieren nur Anfangs- und Endpunkt; erstes Werkzeug und Raumschiff markieren den Weg als wichtigste Punkte. Für Hoffnung auf Befreiung durch ein ganz anderes als das Technik-Prinzip eröffnet sich keine Perspektive; im Unbekannten wird immer nur wieder mit dem schon Bekannten konfrontiert ${ }^{6}$. Die Welt bleibt bei aller räumlich-zeitlichen Offenheit begrenzt.

Diesem grandiosen, filmisch perfekten, von der Aussage über den Menschen jedoch enttäuschenden Entwurf von Zukunft aus Vergangenheit und Gegenwart heraus, setzte Andrej Tarkowskij mit „Solaris” (UdSSR 1972) ${ }^{7}$ gewissermaßen eine notwendige, zumindest aber begrüßenswerte Antwort entgegen: In einer Raumstation, von der aus der Planet Solaris erforscht werden soll, begegnen Wissenschaftler ihrer eigenen Vergangenheit, ihrer eigenen Persönlichkeit, den eigenen Gedanken, die sich materialisieren. Diesem psycho-physischen Phänomen sind sie letztlich nicht gewachsen. Wahnsinn und Selbstmord sind Konsequenzen, die sich aus einer Selbstauslieferung an Wissenschaft und Technik ergeben, die sich keine Grenzen setzte. Wenn in Kubricks „2001” die Entwicklung des anthropomorphen Computers „menschlich" mehr als das Schicksal der Astronauten selbst berührt, so wird in „Solaris” wieder an den Menschen selbst erinnert, der nicht in strahlendem Optimismus in seine extraterrestrische Zukunft ausschreitet, sondern nach seiner Berührung mit dieser buchstäblich und mit direktem Situationsbezug zum biblischen Gleichnis als reumütiger Sohn der Erde zu seinem menschlichen Vater kniefällig heimkehrt. „Solaris” erweist sich als geistig wohl gewichtigster filmischer Beitrag im Rahmen des Genres Science-Fiction; er ist ein suggestiver Appell, die Sphäre der menschlichen Welt nicht beliebig zu überschreiten. Gemäß der Erfahrung „Man kann nur das lieben, was man auch verlieren kann", die Raumfahrem bei ihrer Bemühung um Solaris zufällt, gewinnen sie, wie die Zuschauer, aus der Distanziertheit zur Erde und zu sich selbst eine neue, nun tiefere Zuneigung zur Erde und Menschheit. Die Möglichkeit des Überschritts zu einer auch theologischen Perspektive liegt dabei nicht mehr fern. „Solaris” mutet an wie ein letztlich religiöser Versuch der Mahnung an den Menschen, seiner Schöpfungswürde bewußt und treu zu bleiben.

Angesichts derartig profunder Beiträge zum filmischen Raumfahrt-Genre wundert es nicht, daß auch weiterhin derartige Stoffe kultiviert werden. Die menschliche Phantasie kreist um die Möglichkeiten einer Ausweitung des menschlichen Lebensraumes und um die Frage nach Leben außerhalb des Planeten Erde. So fragt mit einigem Ernst Steven Spielberg in seinem Film „Unheimliche Begegnung der dritten Art” (1977) ${ }^{8}$ danach, ob nichtangesichts der desolaten politischen Verfassung unsererErde eine Harmonisierung - nicht Bed rohung - von Geistwesen anderer Planeten denkbar sei. Wurde schon so weit spekuliert, dann ist das „Raumfahrt-Märchen” nicht mehr weit: In den vergangenen drei 
Jahren haben technisch aufwendige Filme von inhaltlich bescheidenem Anspruch um George Lucas' „Krieg der Sterne” (1976) ${ }^{9}$ herum einen märchenhaften Orientierungspunkt gefunden ${ }^{10}$ : Der Ablenkung von den eigenen Kümmernissen wegen, von denen Zukunft nur schwer ableitbar ist, greift man nach den gigantischen Weltraum-Auseinandersetzungen. Der Bezug zur eigenen Wirklichkeit ist damit gänzlich abgeschnitten, da auch gar nicht gewollt.

Eine zweite Gruppe unter Filmentwürfen einer künftigen Welt wird durch ganz „irdische" und nicht technologisch orientierte Visionen bestimmt, die e her dem Horror-Genre zugerechnet werden müssen. Stichworte sind Gewalt und individuelle wie kollektive Bedrohung. Furcht und Entsetzen, die aus Vorgängen durchaus gegenwärtigen $\mathrm{Zu}$ schnitts entwickelt und filmisch dargestellt werden, projizieren eine künftige Welt, die ganz von dieser Erwartung beziehungsweise Befürchtung bestimmt zu sein scheint. Beispiele liefern John Carpenters „Assault - Anschlag bei Nacht” (1976) ${ }^{11}$ und etwa George A Romero mit „Zombie” (1977) ${ }^{12}$. Ersterer Film schildert exzessiv den nächtlichen Überfall einer nicht näher fixierbaren vielköpfigen Jugendbande auf eine fast verlassene Polizeistation, wobei die Perfektion der Anwendung von Feuerwaffen ihre Entsprechung in der Kompromißlosigkeit der Selbstverteidigung der Angegriffenen findet. Furchtbare Perspektive ist die plötzliche und totale Bedrohung. „Zombie” fügt eine irrationale Komponente atavistischer Vorstellungen von Hölle und Erde hinzu: Gemäß einer alten afrikanischen Vorstellung, daß die Toten auf die Erde zurückkehren, wenn die Hölle sie nicht mehr aufnehmen könne(!), breitet Romero das kollektive Entsetzen plan ins Bild gerückten Menschenfressertums durch die Un-Toten vor dem Zuschauer aus. Gegenmittel ist dann auch nur noch die blinde Überlebensgier: Die Welt als Vorstellung des homo hominilupus. So haarsträubend konstruiert Filme solchen Zuschnitts auch erscheinen - sie sind doch ein nachdenklich stimmendes Element bei der Beschäftigung mit den Erwartungen gegenüber einer künftigen Welt: Denn derartige HorrorVisionen haben ihr Publikum und scheinen insofern eine Entsprechung zu finden in Tiefenschichten des individuellen und kollektiven Bewußtseins.

Die Welt von morgen wird - filmisch gesehen - unter massiven Kommunikationsproblemen leiden. Auf der einen Seite kann ein Überangebot an elektron isch vermittelten Informationen und Unterhaltungsstoffen drohen, was angesichts unserer gegenwärtigen medienpolitischen Grundsatzüberlegungen über Kabel-Fernsehen beziehungsweise Satelliten-Fernsehen ja nicht mehr in allzu ferner Zukunft angesiedelt scheint ${ }^{13}$. Auf der anderen Seite geht die Furcht vor einer Welt um, in der zwischenmenschliche Kommunikation nicht mehr ein selbstverständlicher und natürlich ablaufender Vorgang ist. Es droht Sprachlosigkeit im Vermittlungsgefüge von Gefühlen. Ingmar Bergmans, „Schreie und Flüstern" (1972) ${ }^{14}$ zeichnet davon ein erschütterndes Bild. Es droht der Verlust unvermittelter Liebesfähigkeit und der Wärme der emotionalen Bindung innerhalb und außerhalb von Ehe und Familie. Woody Allens „Innenleben” (1978) ${ }^{15}$ lehrt da das Fürchten. Und diese Thematik ist im internationalen Film als Hauptthema oder in Spuren weit verbreitet. Es steht an die totale Entleerung der gesellschaftlichen Konventionen, angesichts deren Hohlheit sich Zusammenbrüche individueller Existenz häufen dürften. Robert Altmans „Eine Hochzeit” (1977) ${ }^{16}$ setzt dieser Vision farcenreich ein beispielhaftes decouvrierendes Filmdenkmal.

Beim Problem des drohenden Verlusts von Kommunikationsfähigkeit ist auch die Nahtstelle zu derjenigen Generation, die man mit Zukunft am unmittelbarsten verbindet, zur Jugend. Nach ihren Aussich ten stellt am radikalsten die Frage Robert Bressons Film „, Der Teufel möglicherweise" (1977) ${ }^{17}$. Angesichts einer zivilisatorischen Gesellschaft, dieihre 
eigenen physischen Lebensgrundlagen in Umwelt und Natur systematisch zerstört, droht der jungen Generation, die keine Machtmittel besitzt, um diesen Prozessen gegenzusteuem, Sinnverlust. Verweigerung kompromißlosen Zuschnitts bis hin zu totaler Apathie, zur Flucht in Drogen oder in den Suizid ist ein bedrückend vorgestelltes Phänomen, das nicht weniger erschreckend dadurch wird, daß die so Reagierenden klargesichtig genug sind, um andere auf die Gefahren aufmerksam zu machen.

Vom Lebensgefühl her sehr ähnlich, wenn auch in der ausgefalteten Erfahrungswelt bescheidener, ist der Film „Die Indianer sind noch fern” der jungen Schweizerin Patricia Moraz (1977) ${ }^{18}$. Auch hier steht am Ende einer nur kurz skizzierten, aber im Umfeld höchst plausibel verdichteten Studie über eine Oberschülerin deren Tod, wobei kaum zweifelhaft bleibt, daß sie ihn selbst suchte. Der Grund dafür liegtin der Kälte der sozialen Bezüge, in der Vergeblichkeit einer Suche nach Verläßlichkeit, nach Zuwendung, nach Zärtlichkeit. Der Kälte des Gefühls entspricht die gewählte Todesart, die offen metaphorisch gewählt wurde: Das Mädchen wird nach einem Spaziergang im winterlichen Wald erfroren aufgefunden.

Eis und Schnee stehen auch in einem anderen Film als die umfassenden Begriffe für die drohende Kälte, an der die menschliche Welt zugrunde zu gehen droht, in Robert Altmans „Quintett” (1979) ${ }^{19}$. Hier sind nur noch einige wenige Menschen übriggeblieben, die „Quintett” als ein Spiel des schonungslosen Überlebenswillens spielen; von einer Spielrunde bleibt nur ein einziger Mensch lebendig zurück, während die anderen umgebracht wurden - nach der Spielregel -, um auf offener Straße von Hunden gefressen zu werden: Eine Parabel auf die entmenschlichte Welt von übermorgen, die Mangel leidet an der Wärme zwischenmenschlicher Bezüge, die „mörderisch” kalt geworden ist.

Vielleicht, so kann man unter Umständen die wichtige Zukunftsgeschichte „Jonas, der im Jahre 2000 fünundzwanzig Jahre alt sein wird" von Alain Tanner (1976) ${ }^{20}$ zusammenfassen, wird der in diesen Jahren Geborene es doch besser haben als seine Eltern gemeint ist nicht wirtschaftliche, sondern seelisch-geistige Prosperität -, die sich einer Welt der Konsumzwänge, des Klassendenkens, der Kommunikationsunfähigkeit, des Materialismus und der Egozentrik entgegenstemmten, damit jedoch nichts erreichen konnten. Wie der alttestamentliche Jonas im Walfisch überlebte, so hat der kleine Jonas seine fünfundzwanzig Jahre lang Zeit, um ausgespieen zu werden in eine Welt, die dann menschlicher und damit bewohnbarer geworden sein sollte. Doch dies zeigt der Film nicht. Er gewinnt seine Utopie aus der Hoffnung gegen alle Erfahrung der jetzt Lebenden.

Daß sich die Welt für Tanner in den wenigen Jahren zwischen diesem und seinem neuen Film „Messidor" (1979) ${ }^{21}$ eher noch mehr abgekühlt hat, macht betroffen. In „Messidor” versuchen zwei beliebige junge Frauen, aus den Zwängen ihrer jeweils engmaschigen Umwelt auszubrechen, um trampend Erfahrungen einer neuen und ungewohnten Selbständigkeit zu gewinnen. Sie scheitern früh und endgültig, weil sie als Außenseiter und verhältnismäßig Schutzlose dem Zugriff der uniformen Mentalität einer perfekt funktionierenden Konsumgesellchaft ausgeliefert werden; nachdem man sie zu mutmaßlichen Terroristinnen per Fernsehsendung landesweit hochzustilisieren versucht hat, verlieren sie ihr psychisches Gleichgewicht. Ein Mann wird - irrtümlich - von ihnen erschossen; am Ende werden sie zu einer absehbar lebenslangen Strafverbüßung abgeführt. Besondere Fatalität wird angesichts dieser Beispiele dadurch deutlich, daß einer noch jungen Generation, die sich noch gar nicht beweisen und bewähren konnte, Scheitern attestiert wird. 
Via Spielfilm in relevanten Ausprägungen wird der Welt von morgen nicht allzuviel Optimismus entgegengebracht. Abgesehen von der Perspektive auch weiter anhaltenden technisch-naturwissenschaftlichen Fortschritts, dessen Ergebnisse immer skeptischer betrachtet werden, auch wenn man mit akzeptablen Beispielen immer noch rechnet, eröffnet sich für die Menschheit - zumindest in der westlichen Welt - kein Ausblick auf ein harmonisch-menschenwürdiges Zusammenleben. Stehen auch weniger wirtschaftliche Probleme an - ganz anders als in den Ländern der Dritten Welt -, so werden die Probleme der zivilisatorischen Errungenschaften den Menschen im Kern seiner Existenz bedrohend treffen, wenn er nicht umdenkt. Dieses ist die allerdings letztlich allen erwähnten Beispielen gemeinsame Komponente, die von nicht geringem Gewicht ist: Die Filme haben appellative Funktion. Ihr Aufforderungscharakter ist nicht zu übersehen. Das künstlerische Medium Film, das durch spezifisch vermittelte Gestaltungsdichte zu besonderer Erlebnisintensität führen kann, weist, wie jedes Kunstwerk, über sich hinaus. Nicht werden Gegenwart und Zukunft dokumentiert, sondern visionär gestaltet. Je dichter und konzentrierter der Film ist, je stärker Betroffenheit ausgelöst wird, desto wahrscheinlicher ist die Hoffnung, daß der zum Denken angeregte Zuschauer Konsequenzen zieht, auf daß die Welt nicht wird, wie befürchtet wurde, sondern so, wie erhofft werden will.

\section{Anmerkungen}

${ }^{1}$ Eine Sonderstellung nimmt Kuba ein, das aufgrund einer streng zentralistischen staatlichen Filmproduktion und eines hohen Ausbildungslevels der in der Filmproduktion Tätigen den Vergleich mit internationalen Standards nicht zu scheuen braucht. Die Stoffe sind allerdings ideologisch fixiert: Welt von morgen ist die perfekte Welt der nach Bewältigung vorhandener Schwierigkeiten erreichbaren sozialistischen Gesellschaft.

2 Dies betrifft insbesondere Produktionen aus der Sowjetunion, Polen, Ungarn und Jugoslawien.

${ }^{3}$ Symptomatisch dafür ist etwa der Anteil amerikanischer Produktionen bei einer umfänglichen Reihe von Science-Fiction-Filmen, die im Fernsehprogramm der ARD 1978/79 ausgestrahlt wurde: Von insgesamt 39 Filmen stammten 29 aus den Vereinigten Staaten [vgl. Arbeitsgemeinschaft der öffentlich-rechtlichen Rundfunkanstalten der Bundesrepublik Deutschland (ARD) (Hrsg.): Spielfilme im Deutschen Fernsehen ARD 1978. Frankfurt 1977. S. 31 fr.].

"Exponenten solcher Strömungen sind etwa „Fahrenheit 451" (Fahrenheit 451) von François Truffaut (Großbritannien 1966) und „DerSchläfer”' (The Sleeper) von Woody Allen(USA 1973).

5 2001: A Space Odyssey, Großbritannien 1968; Regie: Stanley Kubrick; Buch: S. Kubrick und Arthur C. Clarke; Produktion: MGM, 145 Minuten.

6 Vgl. insbesondere Enno Patalas: 2001 - Odyssee im Weltraum, in „Filmkritik”, Frankfurt 9:1968 S. 638-640.

7 Solaris, UdSSR 1972; Regie: Andrej Tarkowskij; Buch: A. Tarkowskij und Friedrich Gorenstein nach einem Roman von Stanislaw Lem; Produktion: Mosfilm; 170 Minuten.

${ }^{8}$ Close Encounters of the Third Kind, USA 1977; Regie und Buch: Steven Spielberg; Produktion: Phillips; 135 Minunten.

${ }^{9}$ Star Wars, USA 1976; Regie und Buch: George Lucas; Produktion: Star War/20th CenturyFox; 121 Minuten.

10 Ein Beispiel ist „Buck Rogers” (Buck Rogers in the 25th Century) USA 1978; Regie: Daniel Haller; Buch: Larson/Steven; Produktion: Universal; 89 Minuten; vgl. auch John Carpenters „Dark Star”, USA 1975. 
11 Assault on Precinct 13, USA 1976; Regie und Buch: John Carpenter; Produktion: CKK; 91 Minuten.

12 Zombie - Dawn of the Dead, ltalien 1977; Regie und Buch: George A. Romero; Produktion: Dario Argento/Alfredo Cuomo; 118 Minuten.

13 Satirisch aufbereitetes Thema in „Network”, USA 1976; Regie: Sidney Lumet; Buch: Paddy Chayefsky; Produktion: Howard Gottfried; 122 Minuten.

14 Viskningar och Rop, Schweden 1972; Regie und Buch: lngmar Bergman; Produktion: Cinematograph AB/Svenska Filminstitutet; 90 Minuten.

15 Interiors, USA 1978; Regie und Buch: Woody Allen; Produktion: United Artists; 92 Minuten.

16 A Wedding, USA 1977; Regie: Robert Altman; Buch: John Considinc, Patricia Resnick, Allan Nicholls, R. Altman; Produktion: Lion's Gate; 125 Minuten.

17 Le Diable Probablement, Frankreich 1977; Regie und Buch: Robert Bresson; Produktion: Sunchild/Société Française de Productions/G.M.F.; 95 Minuten.

18 Les Indiens sont encore loin, Schweiz/Frankreich 1977; Regie und Buch: Patricia Moraz; Produktion: Filmkollektiv Zürich/Les Films 2001; 97 Minuten.

19 Quintett, USA 1978; Regie: Robert Altman; Buch: Frank Barhydt, Patricia Resnick, R Altman; Produktion: Lion's Gate; 118 Minuten.

20 Jonas qui aura 25 ans en l'an 2000, Schweiz/Frankreich 1976; Regie: Alain Tanner; Buch: A. Tanner und John Berger; Produktion: Action/Citel/SFP/SSR; 110 Minuten.

${ }^{21}$ Messidor, Schweiz/Frankreich 1978; Regie und Buch: Alain Tanner; Produktion: Citel/SSR/ Action/Gaumont; 123 Minuten.

\section{SUMMARY}

The feature film, by reason of its technical presuppositions and structures, is particularly able to develop imaginative dimensions. This constantly raises the question, especially in the western hemisphere, of how he sees the world of tomorrow, and the living conditions of future man. Techniques, the collective threat and individual fears, a loss of communicative power and the passivity of the young are some of the subjects for future-orientated productions. New films by Stanley Kubrick, John Carpenter, Robert Altman, Ingmar Bergman, Robert Bresson, Alain Tanner, Sidney Lumet und Patricia Moraz do not develop very positive perspectives in this regard. A serious discussion of these disturbing perspectives and the artistic relevance of certain productions shows that these films are challenging in their character. The response expected from the viewer is that he should contribute towards the building of a better future.

\section{RÉSUMÉ}

En raison de ses conditions techniques, le film se prête particulièrement à développer une dimension visionnaire. Celle-ci se consacre, spécialement dans les pays de l'hémisphère occidental, continuellement à la question du monde de demain, aux conditions de vie de l'homme futur. La technique, les menaces collectives et les peurs individuelles, la perte de la communication, la résignation de la jeunesse sont les thèmes d'avenir importants des principales productions internationales. Par exemple, les nouveaux films de Stanley Kubrik, John Carpenter, Robert Altman, lngmar Bergman, Robert Bresson, Alain Tanner, Sidney Lumet et Patricia Moraz, ne développent pas spécialement des perspectives optimistes. Le sérieux de la discussion avec les perspectives fatalistes et l'importance artistique des admissions filmiques respectives font cependant aussi comprendre que ces perspectives d'avenir ont un caractère appelatif. Le trouble attendu chez le spectateur doit contribuer à travailler aux conditions pour un avenir meilleur. 


\section{RESUMEN}

Por sus caracteristicas técnicas, el cine está especialmente dotado para desarrollar dimensiones visionarias. Sobre todo en los países del hemisferio occidental, esta dimensión se orienta cada vez más hacia el problema del mundo futuro, hacia las condiciones de vida del hombre del mañana. Técnica, amenaza colectiva, miedo individual, pérdida de la comunicación, juventud condenada a la resignación, son temas futuristas predilectos de importantes producciones internacionales. Perspectivas no precisamente optimistas dominan, por ejemplo, nuevas cintas de Stanley Kubrick, John Carpenter, Robert Altman, Ingmar Bergman, Robert Bresson, Alain Tanner, Sidney Lumet y Patricia Moraz. La seriedad con que se aborda la confrontación con las fatales perspectivas y la relevancia de los motivos que inspiran cada película evidencian, por otra parte, que esas perspectivas futuristas tienen el caracter de llamamiento a la conciencia. El impacto que se espera producir en el espectador contribuirá a crear las premisas para un futuro mejor. 